DOI: https://doi.org/10.11144/Javeriana.upsy17-2.pcdp

\title{
¿Y si pensamos la comunidad con derechos? Psicología Comunitaria, derechos y políticas públicas. Una relación compleja ${ }^{*}$
}

\section{And if we Think about the Community with Rights?: Community Psychology, Rights and Public Policies. A Complex Relationship}

Recepción: 15 Junio 2017 | Aprobación: 20 Junio 2017

\author{
Bárbara Olivares-Espinoza a \\ Universidad Santo Tomás, Chile \\ ORCID: http://orcid.org/0000-0001-8239-8910 \\ María InÉs Winkler Müller \\ Universidad de Santiago de Chile, Chile \\ María Isabel Reyes-Espejo \\ Pontificia Universidad Católica de Valparaíso, Chile \\ Héctor Berroeta Torres \\ Universidad de Valparaíso, Chile \\ Maritza Montero Rivas \\ Universidad Central de Venezuela, Venezuela
}

\footnotetext{
a Autor de correspondencia. Correo electrónico: barbaraolivareses@santotomas.cl

Para citar este artículo: Olivares-Espinoza, B., Winkler Müller, M. A., Reyes-Espejo, M. A., Berroeta Torres, H., \& Montero Rivas, M. (2018). iY si pensamos la comunidad con derechos? Psicología Comunitaria, derechos y políticas públicas. Una relación compleja. Universitas Psychologica, 17(2), 1-13. https://doi. org/10.11144/Javeriana.upsy17-2.pcdp
}

\section{RESUMEN}

Este artículo expone resultados derivados de la tercera etapa de una investigación denominada "Ética y Política: ¿Dimensiones olvidadas en la Psicología Comunitaria?", donde se reflexiona en torno a los derechos de las comunidades que participan de intervenciones comunitarias. Se utilizó una metodología cualitativa y se aplicaron técnicas como grupos focales y entrevistas a usuarios de programas sociales de distinta naturaleza. Como resultado, se elaboró un listado de derechos de la comunidad que pueden ser resumidos en: derecho a la información clara y transparente; derecho a la participación y toma de decisiones; derecho al respeto y reconocimiento de la comunidad; derecho a la autonomía de la comunidad, y derecho a recibir intervenciones de calidad.

Palabras clave

ética; psicología comunitaria; derechos; políticas públicas.

\begin{abstract}
This article presents results from the third stage of an investigation called "Ethics and Politics: Dimensions forgotten in Community Psychology?", where he reflects on the rights of communities participating in community interventions. A qualitative methodology was used and applied techniques as focus groups and interviews with users of social programs of different nature. As a result a list of rights of the community that can be summarized in was developed: right to clear and transparent information; right to participation and decision making; right to respect and recognition of the community; right to autonomy of the community; right to receive quality interventions.
\end{abstract}

Keywords

ethics; community psychology; rights; public policy. 
El cambio de milenio ha sido un período caracterizado por la presencia del enfoque comunitario en las políticas públicas chilenas, traducido en el surgimiento de modelos y técnicas propias de la subdisciplina en los dispositivos de intervención de algunos programas (Krause, Jaramillo, Monreal, Carvacho, \& Torres, 2011). Si bien la inserción de la psicología comunitaria (PC) al campo de las políticas sociales ha permitido su consolidación profesional y académica, las condiciones en que ello ha ocurrido han delineado nuevos desafíos y límites para la generación de prácticas comunitarias, que es necesario analizar. En el contexto latinoamericano, reportes previos han destacado que el aumento de proyectos de intervención comunitaria, por ejemplo, en Brasil, no tiene un impacto directo en el fortalecimiento del compromiso político de los psicólogos sociales, ni ha aumentado la participación de las comunidades involucradas, como señala Fátima Quintal de Freitas (2000). Por su parte, Wiesenfeld (2014) analiza el impacto de la participación en los procesos emprendidos en los últimos años en Venezuela, de lo que concluye que si bien se mejoran condiciones de vida, no las transforman ni logran superar temas centrales.

En Chile, este problema se agudiza, ya que la instalación del sistema económico neoliberal ha permeado el diseño y ejecución de la política pública, acentuando ciertas condiciones y generando efectos: disminuye el rol del Estado (función subsidiaria), se liberaliza el mercado en el marco de una economía orientada a la exportación, el comercio internacional y al mercado de capitales, transformando el mercado de trabajo, los principios de protección social y los servicios públicos (Arteaga \& Martuccelli, 2012). Particularmente, en el ámbito de la política social, disminuyen los principios de cobertura universalista, y aumentan programas específicos orientados a la superación de la pobreza, diseñados para no inducir dependencia asistencialista (Instituto de Políticas Públicas Expansivas-UDP, 2009; Molina, 2006).

La revisión de la literatura especializada producida en las últimas dos décadas muestra un amplio consenso respecto a que la expansión y consolidación de la PC chilena se encuentra vinculada a la expansión de políticas públicas en el país (Alfaro \& Zambrano, 2009; Berroeta, Hatibovic, \& Asún, 2012; Alfaro, 2013; Berroeta, 2014). Desde esta perspectiva, se postula que el modelo neoliberal chileno se constituye en un sello que delimita $y$, al mismo tiempo, sostiene las prácticas de la $\mathrm{PC}$ en el contexto actual (Reyes-Espejo, Olivares, Berroeta, \& Winkler, 2015; Olivares \& ReyesEspejo, 2016).

Diversos autores han referido las tensiones producidas por el modelo neoliberal. Es así, como Nelson (2013, p. 213) plantea que “... muchos de los problemas sociales con los cuales la psicología comunitaria trabaja, han sido exacerbados por las políticas neoliberales". En un contexto en que el Estado evita hacerse cargo del gasto social y de crear condiciones para el desarrollo y bienestar ciudadano, los grupos sociales deben encargarse de las responsabilidades que le corresponden a los gobiernos de turno, lo que se muestra consistente con los resultados de investigaciones recientes en diversas latitudes (Harvey, 2005; Prasad, 2006; Schram, Soss, Houser, \& Fording, 2010; Hasenfeld \& Garrow, 2012; De la Maza, 2011; Brady, Schoeneman, \& Sawyer, 2014; Ortiz, 2014; Fuenmayor, 2014).

Así, también la gestión pública basada en la descentralización y privatización de los servicios ha repercutido fuertemente en el tercer sector, coaccionando su potencial crítico y cuestionador del Estado, y convirtiéndolo en un "clienteconsumidor" más, sometido a los principios del mercado (Hasenfeld \& Garrow, 2012). De acuerdo con Brady et al. (2014), el sistema neoliberal ha impactado en la organización comunitaria a través de tres tendencias: 1) la promoción de una práctica basada en la evidencia como un proceso dominante para guiar la acción profesional; 2) la atención disminuida y la tergiversación de los movimientos sociales en el contexto teórico-práctico de la educación de las comunidades, y 3) la creciente profesionalización de la organización comunitaria. En este contexto, esta última ha tendido a "depender" fuertemente de prácticas tecnocientíficas, centradas en formas 
"objetivas" de conocimiento, que se traducen en intervenciones de corte positivista/cuantitativo, que tienden a mantener el statu quo a través de la promoción de reformas menores que fortalecen el individualismo neoliberal.

Dado el contexto reseñado, elaboramos un proyecto de investigación (FONDECYT $\mathrm{n}^{\mathrm{o}}$ 1130638), ejecutado en los años 2013 al 2015, en el que los objetivos principales estuvieron orientados a identificar las dimensiones ontológica, epistemológica, metodológica, ética y política en un conjunto de programas comunitarios y en la formación en PC de un subconjunto de universidades chilenas. Como resultado de dicha indagación, y a partir del material producido, hemos decantado una etapa final, con el objetivo de centrar el análisis en los derechos de las comunidades.

En una primera etapa, la revisión de 101 programas y proyectos pertenecientes a 10 ministerios chilenos durante el año 2013 mostró una franca ausencia, omisión o exclusión de conceptos, principios, valores y prácticas coincidentes con los principios inspiradores de la PC (Reyes-Espejo et al., 2015). Tales resultados fueron coincidentes con los estudios reportados en otros países, lo que demostró que uno de los principales focos de tensión radica en la verticalidad de la relación que establece la política pública con las comunidades intervenidas (Montero, 2010; Rodríguez, 2009; Hasenfeld \& Garrow, 2012; Brady et al., 2014).

En una segunda etapa, indagamos los mismos aspectos a partir de la revisión de 60 mallas curriculares y una posterior selección de 10 programas (sílabos) de la asignatura de $\mathrm{PC}$, de distintas universidades del país, y encontramos que la dimensión mayoritariamente presente en los programas revisados corresponde a la metodológica, pero que se reduce a indicadores muy acotados, tales como asumir un abordaje colectivo de las problemáticas, una distinción de métodos arriba-abajo y abajo-arriba, y una revisión de técnicas participativas a nivel muy general (Olivares, Reyes-Espejo, Berroeta, \& Winkler, 2016).

En este artículo, reportamos los resultados de una tercera etapa, producto de los hallazgos de las dos primeras fases, donde observamos una débil presencia de los principios ético-políticos de la PC, y nos preguntamos cuáles son los derechos que las comunidades deberían tener en este contexto. Con esto, nos interesa destacar que no son solo los profesionales quienes se enfrentan al desafío de bailar con el "monstruo" (Shinn, 2007) cuando ejecutan políticas públicas. La tensión también, y sobre todo, la experimentan los mismos participantes a quienes se dirige la intervención, y son muchas veces estos, quienes reciben programas con deficiencias técnicas y éticas que no están en condiciones o tienen dificultades para rechazar.

Estamos convencidos de que psicólogas y psicólogos comunitarios deben jugar un rol estratégico que haga posible la construcción de nuevas políticas y programas, donde se incorporen nuevos actores a la acción y reflexión, instalando una lógica programática en sintonía con las comunidades y sus demandas. Esto se traduce en reelaborar algunas perspectivas de acción-reflexión que ubiquen en otra posición a las mismas comunidades, permitiendo el despliegue de su capacidad de agencia. Con esto nos referimos al intento de responder a la pregunta que titula este escrito: iy si pensamos a las comunidades con derechos, y de este modo, facilitamos el desarrollo de nuevas posibilidades para la intervención comunitaria?

Una propuesta interesante la formula una de las coautoras de este texto, Montero (2010), quien propone dimensiones que deberían ser consideradas al momento de diseñar e implementar políticas públicas. Destaca la importancia de reconocer que se trabaja con actores sociales en un escenario complejo, donde el conocimiento popular debe ser valorado como una contribución más en la producción de programas y proyectos comunitarios. Propone también, valorar la construcción de conocimiento desde una perspectiva histórica, a través de la creación de una relación dialógica entre expertos y comunidad. Agrega la necesidad de incorporar la investigación "acción participativa" como metodología de intervención, de transformación y de construcción de conocimientos. Un 
elemento de relevancia, y que destacamos ampliamente en los resultados que se presentan más adelante, se relaciona con la necesidad de entender al otro como coautor del conocimiento, y respetar las diferencias culturales, organizativas y de capacidades. Finalmente, la autora reivindica un asunto central en su producción científica, referida a la valoración del carácter político como espacio de ciudadanía (participación, incidencia pública, derechos).

En particular, un esfuerzo inscrito en la misma línea, lo encontramos en el trabajo de una parte del equipo actual realizado en el marco de una investigación previa, donde a partir de un conjunto de datos empíricos, propusimos orientaciones para garantizar la implementación de intervenciones éticas que se traduzcan en deberes mínimos para el actuar profesional, y que inspiren el diseño de cualquier política de tipo comunitario (Winkler, Alvear, Olivares, \& Pasmanik, 2012).

Es necesario profundizar en tales esfuerzos y lograr un lenguaje común comprensible para todos, incluyendo a políticos, profesionales, la ciudadanía interesada y las comunidades organizadas. En esta dirección, es clave explorar las necesidades sentidas, para que cuando no estén incluidas en las políticas públicas, puedan informar y convencer a quienes las producen, para que sean concebidas como urgencias ineludibles. Es importante superar prejuicios y estereotipos existentes en muchos funcionarios, y también en los especialistas provenientes del ámbito académico y de organizaciones intermedias, los que también deben ser superados por los miembros de las comunidades (Montero, 2010).

A partir de todo lo dicho, sostenemos, que es fundamental para que se logre una contribución mutua entre la política pública y la psicología comunitaria, identificar y respetar ciertas condiciones mínimas o derechos de las comunidades cuando se trabaja con las mismas. Sobre esta base, la investigación en psicología comunitaria puede aportar a la toma de decisiones en políticas sociales, mientras que los procesos de intervención comunitaria contribuyen al diseño de políticas más pertinentes a las necesidades de las comunidades, y la participación de estas mejora el sentido y sostenibilidad de las acciones (Montero, 2009; Ornelas, Vargas, \& Duarte, 2010; Rodríguez, 2012; Nelson, 2013; Alfaro, 2013; Wiesenfeld, 2014). Este artículo viene a colaborar en este propósito, al proponer orientaciones concretas, en clave de derechos que buscan instalarse como referente ético y político, para que quienes toman decisiones puedan incluirlas en sus perspectivas de acción desde los diversos ámbitos en que se despliegan las intervenciones comunitarias en la actualidad. Y como este ejercicio no es tarea sencilla, coincidimos con Sánchez (2015) al proponer que no es posible promover todos los valores a la vez y, por tanto, es relevante para efectos prácticos, hacer el esfuerzo de jerarquizarlos, considerando qué valores y en qué medida son relevantes para los sujetos en una situación dada. De este modo, para priorizar los derechos más relevantes, optamos por preguntarles a los mismos actores. El detalle de ese proceso, lo explicamos en el siguiente apartado.

\section{Metodología}

Esta investigación posee un enfoque cualitativo, dado que este es concordante epistemológicamente con la PC, en especial respecto de la valoración de la diversidad, del contexto y la importancia de los sujetos sociales, en referencia a su discurso y accionar (Banyard \& Miller, 1998; Stein \& Mankowski, 2004). Enfatizamos el carácter constructivointerpretativo del proceso de producción científica (González Rey, 2009), mediado por el reconocimiento de procesos situacionales (plano material e histórico) y afectado por las prácticas sociales y el contexto en que estas se producen (Flores, 2010).

En esta fase de la investigación, nos abocamos a la elaboración de un listado de derechos de participantes de programas comunitarios, para lo cual se aplicaron distintas técnicas de producción de información, que se detallarán a continuación 
(ver Tabla 1), distinguiendo a los participantes para cada caso.

\section{Tabla 1}

Técnicas empleadas y participantes seleccionados

\begin{tabular}{llr}
\hline \multicolumn{1}{c}{ Técnica } & \multicolumn{1}{c}{ Participantes } & $\mathbf{N}^{\mathbf{0}}$ \\
\hline 4 Grupos Focales & $\begin{array}{l}\text { Miembros de la Sociedad Chilena de } \\
\text { Psicología Comunitaria }\end{array}$ & 15 \\
& $\begin{array}{l}\text { Participantes de programa comunitario } \\
\text { (con dependencia gubernamental) }\end{array}$ & 7 \\
& $\begin{array}{l}\text { Participantes programa comunitario } \\
\text { (sin dependencia gubernamental) }\end{array}$ & 8 \\
$\begin{array}{l}\text { 1 Entrevista en } \\
\text { profundidad }\end{array}$ & Líder comunitario & 1 \\
\hline Total & & 31 \\
\hline
\end{tabular}

Utilizamos la técnica del grupo focal, donde a partir de preguntas que guían la conversación, se accede a lo vivido por el sujeto en su calidad de actor en una situación determinada. Se trata de una conversación que representa una inserción del sujeto en su cotidianidad, en el que se describe un conjunto de condiciones reales y acciones posibles (Canales, 2006). Una segunda técnica aplicada fue la entrevista en profundidad, que permitió el acceso a las motivaciones, creencias, esquemas de interpretación y modos de significación que los entrevistados tienen respecto a su participación en programas comunitarios (Gainza, 2006).

La información producida a través de estas técnicas fue analizada utilizando los principios de la teoría fundada empíricamente, que trabaja con los datos, fragmentándolos y examinándolos detalladamente, comparándolos entre sí, para descubrir semejanzas y diferencias entre ellos (Strauss \& Corbin, 2002). Se destaca que en la medida en que se producía el análisis, se iba obteniendo información que permitía decidir la selección de participantes, por lo que se asume que nuevos datos dan origen a nuevas explicaciones que, a su vez, requieren de información que se debe rechazar o comprobar, verificar e integrar como parte de la interpretación del problema analizado (Rodríguez, Gil, \& García, 1999). Siguiendo esta idea, dimos inicio a esta fase del estudio, mediante la creación de dos grupos focales simultáneos con los miembros de la Sociedad Chilena de Psicología Comunitaria, a quienes mostramos resultados preliminares para acceder a sus puntos de vista respecto al fenómeno estudiado. Es decir, al rescatar todo el proceso de producción y análisis de la información de las fases previas, se consolidó una síntesis integrativa que constituyó el material a partir del cual se construyó cada grupo focal. La invitación a los miembros de la Sociedad Chilena de Psicología Comunitaria fue pensada como una instancia que nos permitiría acceder a un grupo importante de expertos en el tema, ya que todos son docentes de asignaturas de psicología comunitaria en diversas universidades chilenas de varias regiones del país, o profesionales que trabajan en programas sociales o en organizaciones no gubernamentales que realizan intervenciones comunitarias. De este modo, el acceso a un grupo selecto de psicólogos nos permitió nutrir los resultados a partir de una variedad de experiencias y extensa trayectoria en el campo.

Posterior a dicha elaboración de datos, a partir de un Proyecto de Colaboración Internacional financiado por FONDECYT (proyecto № 11306381), se realizó una jornada de trabajo con la Dra. Maritza Montero, experta venezolana en $\mathrm{PC}$, en la que durante tres días, analizamos la información disponible desde distintas perspectivas teóricas con su asesoría, contribución, y su larga experiencia en el área.

Así, a partir de la propuesta inicial de un listado de derechos de las comunidades, recolectamos información a partir de grupos focales con actores comunitarios (un grupo para participantes de un programa gubernamental, y otro para participantes de un programa sin dependencia gubernamental), a quienes seleccionamos debido a su experiencia como actores centrales del fenómeno analizado. Son ellos quienes, a partir de su historia (de aciertos y desaciertos con las intervenciones implementadas), pueden proponer derechos fundamentales para todo proceso de intervención que pretenda incluirlos en sus acciones de cambio. Con este grupo también expusimos los resultados de las etapas previas, a través de la selección de la información vinculada particularmente al módulo de cartografía de las prácticas comunitarias, y la validación de los datos existentes. 
Adicionalmente, y para profundizar en algunos aspectos que nos permitieran detenernos en derechos priorizados desde distintas experiencias, entrevistamos a un líder comunitario de gran trayectoria en el ámbito social y comunitario con quien construimos en conjunto un listado de derechos que luego triangulamos junto a la información obtenida previamente. ${ }^{\text {a }}$

Durante el proyecto de investigación se cuidaron especialmente los aspectos éticos, tanto en el proceso de consentimiento informado como en el manejo confidencial de la información y, en general, el respeto por los y las participantes. Se obtuvo un certificado de aprobación ética del Comité de Ética Institucional de la Universidad de Santiago de Chile antes del inicio del proyecto y un certificado de seguimiento o auditoría del mismo comité, durante el último año de la investigación.

\section{Resultados}

A continuación, se presentan los resultados finales de esta investigación, organizados en derechos que corresponden a las categorías centrales que estructuran la información producida, y que fueron elaborados luego de tres años de investigación sistemática en este ámbito.

\section{Derecho a la información clara y transparente}

Contar con información verdadera y oportuna es una de las principales preocupaciones que movilizan a la sociedad en la actualidad, sobre todo cuando la información permite tomar decisiones o revertir otras.

El primer derecho que destacamos se relaciona con el acceso a información clara y transparente, donde se pueda garantizar que las comunicaciones e informaciones a la comunidad se transmitan en un lenguaje claro y comprensible para todos y todas.

Toda comunidad, y las personas que la integran, tienen derecho a ser informadas de todos los aspectos centrales de cualquier investigación o intervención que se le proponga.
Ello incluye conocer a las personas, profesionales, actores sociales y funcionarios públicos que actuarán en el proyecto, así como los principios orientadores y características de la institución, las fuentes de financiamiento y la experiencia en proyectos similares. Para ilustrar este aspecto, incluiremos una cita de un líder comunitario que hace referencia directa a este derecho:

"se debe ser como muy claro con la gente... decirles a qué van... y explicarles muy bien... encargar los... la gente de organizaciones comunitarias, sobre todo hoy ... yo creo que cuando se va a hacer una investigación se debe ser claro y decir qué es lo que se va a hacer y además compartir qué resultó después de eso...". (Luis (líder comunitario), comunicación personal, personaln el marco e flujos deesguardar la identidad de la persona.ates e intercambios que se producen en el marco e flujos de, 11 de enero de 2016).

“...es cómo explicitas todo lo que haces, y si a uno le pagan decir 'yo trabajo en esta ONG y punto', uno no tiene que decir cuánto gana ni nada, yo trabajo no más. Yo soy voluntario en esto, entonces ustedes... queremos que nos ayuden, que sean voluntarios o pedirles una ayuda a la organización o personal. Yo creo que en general la comunidad... si ayuda, lo que les molesta es que le pidan cosas y sin saber para qué...”. (Luis (líder comunitario), comunicación personal, personaln el marco e flujos deesguardar la identidad de la persona.ates e intercambios que se producen en el marco e flujos de, 11 de enero de 2016).

Es relevante, en este sentido, que la comunidad conozca a los equipos profesionales, y que estos informen sobre el financiamiento y, si amerita, sobre la afiliación política a la base, pues esto facilita la toma de decisiones y orienta la demanda que la comunidad pueda dirigir a sus contrapartes institucionales.

“... si a la comunidad se le dice en el comienzo, si alguien tiene la capacidad de decir 'mira, yo soy de este lado', que la gente sepa con quién está conversando, claro, porque de repente uno no sabe con quién está trabajando y te lo encuentras en una oficina de... en algún Ministerio, o te lo encuentras en la oficina de una alcaldía o te lo encuentras en una marcha, y eso va 
a ser una sorpresa... pero tú... si conversas con algunos, o con algunos que sepan, los dirigentes van a saber equilibrar la información a la comunidad. Por ejemplo, yo creo que ellos nos van a ayudar independientemente de cómo piensen”. (Luis (líder comunitario), comunicación personal, personaln el marco e flujos deesguardar la identidad de la persona.ates e intercambios que se producen en el marco e flujos de, 11 de enero de 2016).

Del mismo modo, la comunidad tiene derecho a conocer los productos y efectos de la intervención, proyecto o programa y cómo se evalúa. También se debe informar a la comunidad, la existencia de publicaciones, presentaciones en congresos, difusión en medios de comunicación de masas y cuentas públicas, si las hubiese. Sobre este punto, los académicos y profesionales congregados en la Sociedad Chilena de Psicología Comunitaria destacan que a las comunidades:

“... nunca les devuelven las cosas, esto lo he escuchado muchas veces en distintas partes, que van, fueron a hacer un estudio y la gente se entusiasma o coopera, incluso a veces busca datos y todo, y después les quedaron de mandar la información de vuelta y pasan... un año y todavía no saben nada...". (Grupo focal Sociedad PC, Talca, comunicación personal, 29 de agosto de 2015).

Al mismo tiempo, uno de los representantes de la comunidad sostiene que es relevante entregar un respaldo de participación que pueda funcionar como garantía y que permita comunicar a otros sobre lo investigado:

"Si uno trabaja en una investigación me gustaría que haya un documento que ratifique que la comunidad participó, en qué condiciones y ojalá que... si no aparece en el libro cómo participó o en qué consistió, o qué beneficio recibió, que eso se pudiera explicitar en una carta que la organización pueda guardar y que le pueda servir como testimonio en el futuro...". (Luis (líder comunitario), comunicación personal, personaln el marco e flujos deesguardar la identidad de la persona.ates e intercambios que se producen en el marco e flujos de, 11 de enero de 2016).
La información relativa a la permanencia de los profesionales en la comunidad, o a la duración de la intervención se constituye en un aspecto relevante que también es importante transparentar. De este modo, es posible abordar las expectativas y lograr un trabajo en conjunto responsable y negociado.

\section{Derecho a la participación y toma de decisiones}

Uno de los conceptos centrales para la Psicología Comunitaria es, sin duda, la participación. Sabemos que constituye un horizonte al cual aspirar en cualquier proceso de intervención comunitaria. Es por esto, que se propone que la comunidad tiene derecho a aceptar o rechazar las propuestas de intervención, de modo de que no se les imponga iniciativas desde fuera, o no se considere sus necesidades o intereses. Ello implica aprobar o rechazar cada una de las etapas, desde el diseño hasta la difusión de sus resultados.

En este sentido, uno de los miembros de la Sociedad Chilena de Psicología Comunitaria destaca, en este punto, lo relevante que resulta que las comunidades puedan decidir respecto a su propia participación en las iniciativas comunitarias a las que son convocadas:

"Como muchas veces algunos programas o
intervenciones intentan abarcar a la mayoría
de los usuarios de la comunidad, o a todos
los usuarios de las comunidades... darle la
oportunidad o el derecho a alguna persona de
la comunidad a negarse a esa intervención, en
el entendido de que puede ser una actividad de
control social, de acuerdo a su particularidad
cultural. Yo estoy pensando sobre todo en
contextos rurales o indígenas en donde los
programas funcionan más como mecanismos de
control, que como mecanismos de liberación
o de desarrollo comunitario. Entonces, negarse
también podría ser una posibilidad... como
habitualmente se utiliza en los consentimientos
informados en investigación...". (Grupo focal
Sociedad PC, Talca, comunicación personal, 29
de agosto de 2015).

La participación es un proceso que se desarrolla en el tiempo. No solo se necesita tener la oportunidad de participar, sino también contar 
con acompañamiento técnico para desarrollar las capacidades que la permiten. En este sentido, uno de los miembros de la comunidad enfatiza cómo fue su experiencia de participación en un programa gubernamental, donde el equipo ejecutor acompañó y facilitó la toma de decisiones a lo largo del proceso:

"entonces, ahí nos explicaron... cómo eran las fases... en qué... en qué... cómo... o sea, el paso a paso de todo este proceso... y dentro de eso también, estaba el plan de qué se decidió como... porque nos iban a dar una cantidad de dinero... cierto, y que teníamos que saber un poco... decidir entre todas esas... claro... priorizar entre varias opciones que fuimos dando...”. (Grupo focal, organización con dependencia gubernamental, comunicación personal, 09 de diciembre, 2015).

\section{Derecho al respeto y reconocimiento de la comunidad}

El respeto y el reconocimiento son valores universales que forman parte del contrato social que nos organiza actualmente como sociedad. En concreto, se propone que la comunidad tiene derecho a que cualquier proyecto $\mathrm{o}$ programa sea pertinente a su contexto y que se consideren y respeten experiencias previas. De este modo, las comunidades destacan lo oportuno que puede resultar, que los equipos conozcan con anticipación los contextos en los que se proponen intervenir, rescatando antecedentes que permiten comprender la historia de cada lugar:

“... investigaron y no llegaron con... a preguntar lo mismo de siempre: para qué, si por qué; no, si no que con un punto... validaron solamente cosas que ellas tenían claras... investigaron y nos trajeron propuestas bastante claras de lo que nosotros habíamos dicho muchas veces...". (Grupo focal, organización sin dependencia gubernamental, comunicación personal, 05 diciembre, 2015).

En este mismo ámbito, la comunidad tiene derecho a que se conozcan y respeten sus historias, prácticas y saberes, así como también sus tiempos. Es necesario que se construya una relación o vínculo con la comunidad antes de intervenir, y que los agentes externos se familiaricen con la comunidad y sus integrantes, de forma que se minimice el riesgo de daños a la comunidad.

"yo creo que debieran incorporarse como uno más... en un período de tiempo antes de comenzar un trabajo ir un par de veces a conocer qué pasa, a conocer la gente... bueno en una población uno tiene mayor capacidad de observar algunas cosas que le van a permitir entrar con mayor facilidad después... pero cuando uno llega de repente así... y quiere hacer la charla o dar un taller o hacer el curso... va a depender del estado de ánimo de la gente... si no lo conocen es probable que le crean o no le crean... pero si ya lo han visto antes... y lo sienten como uno más del... va a ser todo más fácil...”. (Luis (líder comunitario), comunicación personal, personaln el marco e flujos deesguardar la identidad de la persona.ates e intercambios que se producen en el marco e flujos de, 11 de enero de 2016).

\section{Derecho a la autonomía de la comunidad}

La autonomía constituye un pilar esencial para todo proceso de intervención que busque el fortalecimiento comunitario. Es así como toda comunidad tiene derecho a que la intervención desarrolle capacidades necesarias para hacerse cargo de mantener los logros de la intervención, monitorear y hacer seguimiento de la continuidad de los proyectos. La comunidad no se debe sentir presionada a aceptar alguna propuesta de intervención sin antes evaluar y comprender su utilidad y alcance.

"Nosotros tenemos que aceptarlo, porque no se puede hacer otra cosa, así que... y llegan con un programa, le ofrecen a usted por ejemplo, ya, vamos a traer tal cosa y... no sé, después uno queda esperando... porque no tenemos programas...”. (Manuel (adulto mayor), organización sin dependencia gubernamental, comunicación personal, 27 de noviembre de 2015). 


\section{Derecho a recibir intervenciones de calidad}

Las comunidades tienen derecho a que las intervenciones, proyectos o programas que se les ofrecen, estén bien elaborados, se sustenten en datos y evidencias comprobables y consideren los recursos materiales y humanos necesarios para su adecuada implementación. El reverso de este derecho, lo constituyen las experiencias que solo se limitan a proyectos de corto plazo que prometen un cierto beneficio y finalmente, no logran concretar los productos comprometidos. Un testimonio de ello, lo relata un participante de un programa comunitario:

"era un programa en que a nosotros nos citaban y... yo asistí como a 3 ó 4 reuniones, pero me fui dando cuenta de que cada vez iba menos gente... y las cosas no se veían... y no se seguía una continuidad... me entiendes... entonces nos íbamos quedando en el tiempo y no se veía nada... o sea, de todo lo que se pedía, de todo lo que se hablaba, nada llegaba a término...". (Grupo focal, organización con dependencia gubernamental, comunicación personal, 09 diciembre, 2015).

Las intervenciones de calidad en el campo de lo comunitario requieren de mínimos que se condicionan también a partir de los derechos descritos previamente. Particularmente cuando se trata del trabajo comunitario, la calidad también pasa por dar espacios de participación, promover la autonomía y reconocer los deberes y derechos de los participantes, es decir, mostrar cierta coherencia con principios y valores que enmarcan el trabajo comunitario. En este contexto, podemos observar la siguiente cita:

"O sea como mínimo hacerse cargo de que uno dice que va a trabajar con horizontalidad, que va a trabajar con la participación, mínimo, o sea para un decálogo debería ser lo mínimo, que de verdad respete eso. Yo me acuerdo de un psiquiatra que decía, de la [universidad] católica, él decía, que es el director de una unidad de corta estadía que... Me decía a mí nunca nadie me habló de lo comunitario, o sea yo no tenía idea hasta que no llegué a trabajar, empecé a ver necesito las redes, necesitamos trabajar entre todos digamos, y me decía, al final yo siento que esto es como una actitud, es una actitud que yo debo tener". (Grupo focal Sociedad PC, Talca, comunicación personal, 29 de agosto, 2015).

En síntesis, los resultados presentados dan cuenta de la elaboración de un listado de cinco derechos que toda intervención comunitaria, incluyendo los programas de las políticas públicas, deberían considerar y respetar. Todos ellos remiten a un derecho básico, que corresponde al respeto por el Otro. Los derechos a la información, a la participación, al respeto, la autonomía y a la calidad, pueden ser entendidos como formas o manifestaciones de una actitud de respeto, en que se reconoce en el otro un igual, al que corresponde escuchar y validar.

\section{Discusión}

Los resultados que acabamos de presentar constituyen un ejercicio de memoria, que busca volver a centrar el foco en las comunidades, y recuperar el espíritu fundante de la PC, que se planteó en ese entonces, como una psicología de la acción para la transformación, que tiene como característica esencial que lo comunitario incluye el rol activo de la comunidad, es decir, su participación. Y no solo como invitada, o como espectadora aceptada o receptora de beneficios, sino como agente activo con voz, voto y veto (Montero, 2004); esto es, una comunidad con derechos.

Volver a pensar una comunidad con derechos implica detenerse en aquello que la fortalece y la convierte en actor clave del proceso. Sin embargo, considerando el contexto en el que actualmente se produce política pública (Alfaro \& Zambrano, 2009; Berroeta et al., 2012; Alfaro, 2013; Berroeta, 2014), la noción de comunidad se ve atravesada por múltiples interferencias que bloquean su alcance emancipador, mercantilizan lo común y restan capacidad para negociar y cuestionar las relaciones que reproducen el sometimiento de amplios sectores de la población. Las políticas sociales altamente focalizadas construyen un sujeto carente y que, habitualmente, como efecto de la intervención, 
se siente agradecido de la acción recibida. El agente externo, por su parte, queda instalado en un lugar de ayuda, despolitizado, y con escasa capacidad para incidir en el mejoramiento de la vida de las comunidades atendidas (Montenegro, Rodríguez, \& Pujol, 2014). De este modo, pensar una comunidad con derechos no es solo un desafío para los miembros de la comunidad, sino para los psicólogos comunitarios, y también para la propia disciplina que se enfrenta constantemente a reelaborar el horizonte disciplinar hacia el cual encamina su accionar (Wiesenfeld, 2006).

Reconocer el derecho a la participación y toma de decisiones por parte de la comunidad es central en los procesos de intervención comunitaria que se desarrollan en la actualidad. La experiencia latinoamericana reporta que desde las políticas públicas, se promueven modos de participación a partir de parámetros predefinidos, e incluso, se ha señalado que se ha utilizado con frecuencia como herramienta de cooptación ideológica y cultural de líderes y organizaciones en general, a pesar de haber declarado genuinamente que se promovía una participación comunitaria (Rodríguez, 2009). De esta forma, la participación muchas veces se traduce como 'expresión de puntos de vista' o como recibir algo a cambio de valorar positivamente y adherirse a una política pública. Tal y como plantea uno de los entrevistados, los informantes clave que deberían facilitar la participación comunitaria, se transforman en delegados de políticas territoriales, lo que genera la captura estatal de formas informales de participación y el consecuente debilitamiento de su potencial transformador (Montenegro et al., 2014).

El derecho a la autonomía y a recibir intervenciones de calidad permite resguardar que los diseños consideren tiempos respetuosos con los procesos emprendidos. Muchos de los programas públicos son dirigidos desde afuera hacia adentro, o de arriba hacia abajo, sin informar, ni sensibilizar a la comunidad respecto de la solución o cambio propuesto (Rodríguez, 2009; Hasenfeld \& Garrow, 2012; Brady et al., 2014). En su mayoría, funcionan mientras los agentes externos están a cargo, pero cuando estos se retiran, suelen cerrarse y se terminan perdiendo los aprendizajes derivados del proceso de intervención (Montero, 2010).

Ya en 1959, Orlando Fals Borda proponía el principio de la autonomía del grupo, y señala que toda acción debe ser decidida, organizada y realizada con una orientación democrática, por y con los grupos organizados de la comunidad, lo que coincide con lo propuesto por los participantes consultados. Acá debemos detenernos en lo relevante que resulta entender a las comunidades como entidades dinámicas, influyentes en su propio destino, cuyos conocimientos y experiencias deben ser escuchados. Al mismo tiempo, se requiere que los profesionales que trabajen con estas comunidades, se consideren facilitadores y copartícipes de los procesos de cambio, estableciendo una relación simétrica, horizontal, de respeto mutuo, donde se compartan saberes, afectos y experiencias (Wiesenfeld, 2006).

Respecto al derecho a intervenciones de calidad, adquiere especial relevancia en el contexto de la formación en psicología comunitaria, ya que cuando se dictan asignaturas en la subdisciplina, con cierta frecuencia, se solicita o instruye a los y las estudiantes para que realicen "experiencias de campo" cuya calidad puede ser puesta en cuestión. Los tiempos de la academia (Montero \& Giuliani, 1999; Olivares et al., 2016) están centrados en los procesos de aprendizaje de los estudiantes, y no consideran en forma suficiente los ritmos y necesidades de las comunidades. El equilibrio entre promover bienestar en las comunidades y constituirse en instancias poderosas de aprendizaje es precario y con cierta facilidad puede tornarse en riesgos para las comunidades.

Consideramos que es sobre la base de asegurar estos derechos mínimos de las comunidades en las intervenciones, que se realizan al alero del Estado, que es posible una contribución mutua entre la política pública y la psicología comunitaria, que acerca posiciones y diluye tensiones que han afectado la actuación profesional desde hace décadas. 


\section{Agradecimientos}

Por Maritza Montero:

Agradezco a María Inés Winkler Müller, a María Isabel Reyes Espejo, a Bárbara Olivares y a Hector Berroeta, el haberme invitado a participar en este trabajo, que para mí culmina algo fundamental en el trabajo comunitario, que permite hacer oficiales y jurídicos, derechos que deben hacerse conocer, no solo en Chile, sino en todos los países latinoamericanos.

\section{Referencias}

Alfaro, J., \& Zambrano, A. (2009). Psicología Comunitaria y políticas sociales en Chile. Revista Psicología $\mathbb{E}$ Sociedade, 21(2), 275-282. Recuperado de http://www.scielo. br/pdf/psoc/v21n2/v21n2a15

Alfaro, J. (2013). Psicología Comunitaria y Políticas Sociales: Institucionalidad y dinámicas de actores. Global Journal of Community Psychology Practice, 4(2). Recuperado de http://www.gjcpp.org/pdfs/a lfaro-v4i2-20130613.pdf

Arteaga, C., \& Martuccelli, D. (2012). Neoliberalismo, corporativismo y experiencias posicionales: Los casos de Chile y Francia. Revista mexicana de sociología, 74(2), 275-302.

Banyard, V., \& Miller, K. (1998). The powerful potencial of qualitative research for community psychology. American Journal of Community Psychology, 26(4), 485-505.

Berroeta, H., Hatibovic Diaz, F., \& Asún Salazar, D. (2012). Psicología Comunitaria: prácticas en Valparaíso y visión disciplinar de los académicos nacionales. Polis, Revista Latinoamericana, 11(31), 335-354.

Berroeta, H. (2014). El quehacer de la Psicología Comunitaria: coordenadas para una cartografía. Psicoperspectivas, 14(2), 19-31.

Brady, S., Schoeneman, A., \& Sawyer, J. (2014). Critiquing and Analyzing the Effects of Neoliberalism on Community Organizing: Implications and Recommendations for
Practitioners and Educators. Journal for Social Action in Counseling and Psychology, 6(1), 36-60.

Canales, M. (2006). Metodologías de investigación social. Introducción a los oficios. Santiago de Chile: LOM.

De la Maza, G. (2011). Espacio público y participación ciudadana en la gestión pública en Chile: límites y posibilidades. Polis, 10(30), 45-75.

Fals-Borda, O. (1959). Acción comunal en una vereda colombiana. Bogotá: Universidad Nacional de Colombia.

Flores, R. (2010). Observando Observadores. Santiago de Chile: Ediciones UC.

Fuenmayor, J. (2014). Política pública en América Latina en un contexto neoliberal: Una revisión crítica de sus enfoques, teorías y modelos. Cinta de Moebio, 50, 39-52.

Gainza, A. (2006). La entrevista en profundidad individual. En M. Canales (Ed.), Metodologías de la investigación social (pp. 219-256). Santiago de Chile: LOM.

González Rey, F. (2009). Epistemología y Ontología: un debate necesario para la psicología de hoy. Revista Diversitas Perspectivas en Psicología, 5(2), 205-224.

Harvey, D. (2005). A Brief History of Neoliberalism. Oxford: Oxford University Press.

Hasenfeld, Y., \& Garrow, E. (2012). Nonprofit Human-Service Organizations, Social Rights and Advocacy in Neoliberal Welfare State. Social Service Review, 86(2), 295-322.

Instituto de Políticas Públicas Expansivas-UDP. (2009). El Chile que viene. De dónde venimos, dónde estamos y a dónde vamos. Santiago de Chile: Ediciones UDP.

Krause, M., Jaramillo, A., Monreal, V., Carvacho, H., \& Torres, H. (2011). Historia de la Psicología Comunitaria en Chile: desde la clandestinidad a la política pública. En M. Montero, \& I. SerranoGarcía (Comps.), Historias de la Psicología Comunitaria en América Latina. Participación y Transformación (pp. 115-138). Buenos Aires: Paidós. 
Molina, C. (2006). Universalismo básico. Una nueva política social para América Latina. Washington, D.C.: Interamerican Development Bank.

Montenegro, M., Rodríguez, A., \& Pujol, J. (2014). La Psicología Social Comunitaria ante los cambios en la sociedad contemporánea: De la reificación de lo común a la articulación de las diferencias. Psicoperspectivas, 13(2), 32-43.

Montero, M., \& Guiliani, F. (1999). La docencia en psicología social comunitaria: algunos problemas. Psykhe, 8(1), 57-63.

Montero, M. (2004). Introducción a la Psicología Comunitaria. Buenos Aires. Paidós.

Montero, M. (2009). Community Action and Research as Citizenship Construction. American Journal of Community Psychology, 43(1-2), 149-161.

Montero, M. (2010). Unión de actores sociales, participación comunitaria y ética, en la ejecución de políticas públicas. En A. Hincapié (Comp.), Sujetos políticos y acción comunitaria. Claves para una praxis de la psicología social y de la clínica social-comunitaria en América Latina (pp. 45-66). Medellín: Universidad Pontificia Bolivariana.

Nelson, G. (2013). Community Psychology and Transformative Policy Change in the Neoliberal Era. American Journal of Community Psychology, 52 (3-4), 211-223.

Olivares, B., \& Reyes-Espejo, M. I. (2016). Evaluación de un programa social en infancia desde los principios de la Psicología Comunitaria. Revista Latinoamericana de Ciencias Sociales, Niñez y Juventud, 14(1), 431-444.

Olivares, B., Reyes-Espejo, M. I., Berroeta, H., \& Winkler, M. I. (2016). La Formación Universitaria en la Psicología Comunitaria Chilena de Hoy: iUn Lugar Subalterno? Psykhe, 25(2), 1-12.

Ornelas, J., Vargas-Moniz, M., \& Duarte, T. (2010). Community Psychology and Social Change: A Story from field of Mental Health in Portugal. Global Journal of Community Psychology Practice, 1 (1), 21-31.
Ortiz, M. (2014). El perfil del ciudadano neoliberal: la ciudadanía de la autogestión neoliberal. Sociológica, 29(83), 165-200.

Prasad, M. (2006). The Politics of Free Markets: The Rise of Neoliberal Economic Policies in Britain, France, Germany, and the United States. Chicago, IL: University of Chicago Press.

Quintal de Freitas, M. (2000). Voices from the South: The Construction of Brazilian Community Social Psychology. Journal of Community $\mathbb{E}$ Applied Social Psychology, 10(4), 315-326.

Reyes-Espejo, M. I., Olivares, B., Berroeta, H., \& Winkler, M. I. (2015). Del discurso a las prácticas: Políticas sociales y psicología comunitaria en Chile. Polis, Revista Latinoamericana, 42. Recuperado de https://polis.revues.org/11413

Rodríguez, A. (2009). Social Policies in Uruguay: A view from the political dimension of Community Psychology. Journal of Community Psychology, 43 (1-2), 122-133.

Rodríguez, A. (2012). Aportes de la psicología comunitaria al campo de las políticas públicas sociales: el caso de Uruguay. En J. Alfaro, A. Sánchez, \& A. Zambrano (Eds.), Psicología Comunitaria y Políticas Sociales. Reflexiones y experiencias (pp. 111-142), Buenos Aires: Paidós.

Rodríguez, G., Gil, J., \& García, E. (1999). Metodología de la Investigación Cualitativa. Granada: Aljibe.

Sánchez, A. (2015). 'Nuevos' valores en la práctica psicosocial y comunitaria: Autonomía compartida, auto-cuidado, desarrollo humano, empoderamiento y justicia social. Universitas Psychologica, 14(4), 15-24.

Schram, S., Soss, J., Houser, L., \& Fording, R. (2010). The Third Level of US Welfare Reform: Governm entality under Neoliberal Paternalism. Citizenship Studies, 14(6) 739-754. Recuperado de http://www.bama.ua.edu/ rcfording/ss hfcitizenshipstudies.pdf 
Shinn, M. (2007). Waltzing with a Monster: Bringing Research to Bear on Public Policy. Journal of Social Issues, 63(1), 215-231.

Stein, C., \& Mankowski, E. (2004). Asking, witnessing, interpreting, knowing: conducting qualitative research in community psychology. American Journal of Community Psychology, 33(1-2), 21-35.

Strauss, A., \& Corbin, J. (2002). Bases de la investigación cualitativa. Técnicas y procedimientos para desarrollar la teoría fundamentada. Medellín: Universidad de Antioquia.

Wiesenfeld, E. (2006). El rescate de las comunidades en el marco de la Globalización. Athenea Digital, 9, 46-57.

Wiesenfeld, E. (2014). La Psicología Social Comunitaria en América Latina: ¿Consolidación o crisis? Psicoperspectivas, 13(2), 6-18.

Winkler, M., Alvear, K., Olivares, B., \& Pasmanik, D. (2012). Querer no Basta: Deberes Éticos en la Práctica, Formación e Investigación en Psicología Comunitaria. Psykhe, 21(1), 115-129.

\section{Notas}

* Artículo de investigación. Sintetiza los principales resultados derivados de la investigación denominada "Ética y Política: ¿Dimensiones olvidadas en la Psicología Comunitaria?”, № 1130638, que contó con el financiamiento de la Comisión Nacional de Investigación Científica y Tecnológica de Chile, con fecha de inicio el 5 de Marzo de 2013 hasta 15 de Marzo de 2016 y el patrocinio de la Universidad de Santiago de Chile y la Universidad de Valparaíso.

a Para acceder al detalle de los resultados, puede consultar el siguiente artículo, recientemente publicado: Reyes, M. I., Olivares, B., Berroeta, H., \& Winkler, M. I. (2016). Del discurso a las prácticas: Políticas sociales y psicología comunitaria en Chile. POLIS, 42. Otros datos son presentados en el manuscrito titulado "Community psychology, public politics and neoliberalism: the chilean experience" (en proceso de revisión). 